\title{
Protein Truncation Abnormality
}

National Cancer Institute

\section{Source}

National Cancer Institute. Protein Truncation Abnormality. NCI Thesaurus. Code C45596.

Translation of a truncated form of a wild-type protein that originates from a mutation or mutations in the coding sequence of a gene. Genetic alterations that yield truncated protein products include frameshift mutations, nonsense mutations or splice site mutations. The activity of a truncated protein is usually reduced or abolished in comparison to the activity of the wild-type protein. 